\title{
HISTOLOGICAL STUDY OF REPARATIVE CAPACITY OF SILVER NANOPARTICLES ON AGE RELATED CHANGES OF ALVEOLAR BONE, CEMENTUM AND PERIODONTAL LIGAMENT IN RATS
}

\author{
Tahany Haggag* and Elham F. Mahmoud**
}

\begin{abstract}
Background: Nanotechnology is a rapidly growing scope in recent research, it has a widely acceptable range of potential implications in humans, particularly for dentistry. Among various metallic nanoparticles, silver nanoparticles (AgNPs) are considered the most animated and charming nanoparticles that are involved in multiple biomedical applications.

Aim: The goal of the current study was to determine the possible biological reparative influences of silver nanoparticles on age changes of the alveolar bone, cementum and periodontal ligaments of rats

Material and methods: Forty five male albino rats (fifteen adults aging six months and thirty old aging eighteen months) were divided equally into three groups. Group I (adult age) that get infused with saline intravenously to serve as control group, group II (old age) which also get infused with saline intravenously and group III (old age received an oral dose of silver nanoparticles $100 \mathrm{mg} / \mathrm{kg}$ ) once daily for 4 weeks.

Results: Old age group revealed sever histological alterations as thinning of bone trabeculae and widening of bone marrow cavities. Marked hypercementosis with multiple signs of degeneration in cementum, periodontal ligaments (PDL) and alveolar bone were evident, while old group received silver nanoparticles revealed a considerable improvement of histological features of the tissues under investigation which became nearly similar to that of control group. DEXA examination revealed statistical significant difference in the alveolar bone mineral density between different groups.
\end{abstract}

Conclusion: Oral administration of silver nanoparticles considerably ameliorated most of histological features of alveolar bone, cementum and periodontal ligaments of old rats. Bone mineral density of old rats was improved by silver nanoparticles.

KEYWORDS: Aging; Cementum; Periodontal ligaments; Rats; DEXA.

* Associate Professor, Department of Oral Biology, Faculty of Oral and Dental Medicine, Cairo University, Egypt

** Associate Professor, Department of Oral Biology, Faculty of Oral and Dental Medicine, Suez Canal University, Egypt 


\section{INTRODUCTION}

Nanoscience and nanotechnology have been developed in the bio-manufacturing of metal nanoparticles (MNPs). The MNPs are widely applied in material sciences, chemistry, catalysis, medicine, biology and biotechnology ${ }^{(1)}$. Nanotechnology is an outflowing field for the current research and has been advanced in various disciplines. There are four classes of nanomaterials such as metals, polymers, composite and ceramic, all these have been developed for potential application in health care $^{(2)}$.

Many nanoparticles have been added to materials used in dentistry. Among these applications, silver nanoparticles being incorporated into the existing materials such as dental filling agents, adhesive, and acrylic denture base. The improved chemical structure of silver nanoparticles make it to be characterized by good antiwear, antibacterial, and anti-fungicidal properties ${ }^{(3)}$.

Multiple factors are affecting the biological activity of AgNPs such as particle size, surface chemistry, particle morphology, distribution shape, particle components and dissolution rate. The determination of cytotoxicity of AgNPs depends on particle reactivity in solution, efficiency of ion release and the type of reducing agents utilized for the synthesis of AgNPs . ${ }^{(4)}$.

AgNPs with a diameter particle size less than $20 \mathrm{~nm}$ are effective in the treatment of particular infectious disorders ${ }^{(5)}$ and is efficient in delaying the bacterial and mold growth as well as deleterious spores. Atiyeh et al., ${ }^{(6)}$ reported that silver is an applicable and efficient treatment choice for infections associated burns, open wounds, and chronic ulcers.

Most of the scientific studies that evaluate the biological effects of nanomaterials were in vitro, while few in vivo studies were carried out for the same purpose ${ }^{(7)}$. Latterly, there is more attention for the broad use of nanoparticles and its potential dangers. Incomplete estimation on silver nanoparticle cytotoxicity is responsible for insufficient regulatory control over the utilization or elimination of such products of AgNPs ${ }^{(8)}$.

Ageing is an uncompromising process, which leads to disturbance of homeostasis, reduction in adaptation to environmental stimuli, affecting cells, tissues, organs and bodily systems. In addition to an increased susceptibility to illness and death. Controversial continues as to whether the physiological and pathological effects characterizing ageing are due to the ageing process itself or to disorders, medicine, or environmental alterations to which old people are exposed ${ }^{(9)}$.

The oral health of the aging people is, in part, problematic, as most of them have or require some form of dental prostheses with high incidence of periodontal disorders and root caries. Quality of life of the elderly was directly influenced by this trend, causing psychological, physical and social harm to these patients ${ }^{(10)}$. Therefore, the goal of the current study was to determine of the impacts of oral administration of silver nanoparticles on age changes of alveolar bone, cementum and periodontal ligaments of rats.

\section{MATERIALS AND METHODS}

Forty five male albino rats (fifteen adults aging six months and thirty olds aging eighteen months), weighing 100-150 grams each, were get from the "Animal Research center" Faculty of medicine, Cairo University, were used in this study. The rats were kept in specially designed wire cages and were maintained under good ventilation. They fed standard laboratory diet and supplied drinking water ad-libitum. The animals were divided into three equal groups, fifteen rats for each group. Group I (adult group) get infused with saline intravenously to serve as control group, Group II (old age group) get infused with saline intravenously and 
group III, old rats received a daily oral dose of silver nanoparticles $(100 \mathrm{mg} / \mathrm{kg})$ for 4 weeks.

\section{Preparation of silver nanoparticles}

Preparation of 5x10-3 mol/l of Ag nanospheres (puress, Fluka) was done by citrate-reduction route (11) according to the following: 0.0850 grams of silver nitrates (AgNO3) (puress, Fluka) were added to 100 $\mathrm{ml}$ of double distilled water. Thereafter, we added $25 \mathrm{ml}$ of the stock solution to $100 \mathrm{ml}$ of double distilled water. Then, we heated the solution to begin to boil where $5 \mathrm{ml}$ of $1 \%$ of sodium citrate was added with potent magnetic stirring. We continued heating until we observed the gradual change in color of the solution into yellow. Subsequently, we continued heating for another 15 minutes after that, we removed the solution from the heater and stirred for another 15 minutes. We completed the silver nanoparticles solution to $125 \mathrm{ml}$ by adding double distilled water and stored at $4{ }^{\circ} \mathrm{C}$.

\section{Specimen Preparation and Histopathological Examination}

We dissected out the lower jaws and separated it into halves (right and left sides). We fixed the right sides of the mandibles in $10 \%$ formaldehyde solution for $24 \mathrm{hrs}$. Then we decalcified it in 15\% ethylene diamine tetra-acetic acid (EDTA), for 28 days at $4^{\circ} \mathrm{C}$. After decalcification was finished, the mandibles were rinsed with buffer solution, dehydrated in ethyl alcohol, cleared in xylol and embedded in paraffin wax. Sagittal sections of 4-6 um were cut with rotary microtome, mounted on glass slides, stained with Haematoxylin and Eosin stain and examined under light the microscopic.

\section{Alveolar bone mineral density measurement by DEXA}

Left sides of the mandibles were stored in normal saline and used for measurement of bone mineral density (BMD) of alveolar bone. The jaws were dried prior to radiographic examination.
Alveolar bone mineral density was measured at points opposite to alveolar bone supporting the root apices of the first molars by Dual Energy X-ray absorbometery (DEXA) using Norland XR-46 at the Unit of Bone Mineral Density at National Research Center. Results are expressed as BMD (g/ cm2). Statistical analysis was performed using analysis of variance ANOVA test to compare mean of alveolar bone mineral density between different groups.

\section{RESULTS}

\section{Group I (Control group)}

Light microscopic examination of alveolar process of control group revealed that, its compact bone consists of Haversian systems which is formed of Haversian canals surrounded by concentric and interstitial lamellae of bone. Well-developed osteocytes were observed (Fig. 1). In the same group, oblique fibers of periodontal ligaments (PDL), inter-radicular and apical fibers were characterized by well-organized orientation. Normal density of fibroblasts and collagen fibers were noted. The fibers occupied the normal width extending between cementum and the alveolar bone (Figs 2, 3\& 4).

\section{Group II (Old age group)}

Light microscopic examination of alveolar bone of old group exhibited thinning, irregularity of bone trabeculae and widening of bone marrow cavities. Bone marrow cavities contained numerous fat cells. Some empty lacunae of osteocytes were observed (Fig.5). Disorganization of oblique fibers of PDL and fibroblasts in some areas were noted with widening of interstitial spaces. Resorption and irregularity of adjacent bone and cementum surfaces were evident (Fig.6). Loss of normal arrangement of interradicular group of PDL with areas of degeneration and large dilated blood vessels have been revealed. Thickened, irregular inter-radicular cementum and resorbed bone surface were evident (Fig.7). Apical group appeared deformed, amalgamated 
and disoriented with signs of degeneration. Marked hypercementosis was noted (Fig.8).

\section{Group III (Old age group received Nano silver):}

Light microscopic examination of alveolar bone of group III as compared with the control group, it revealed increased thickness of bone trabeculae with

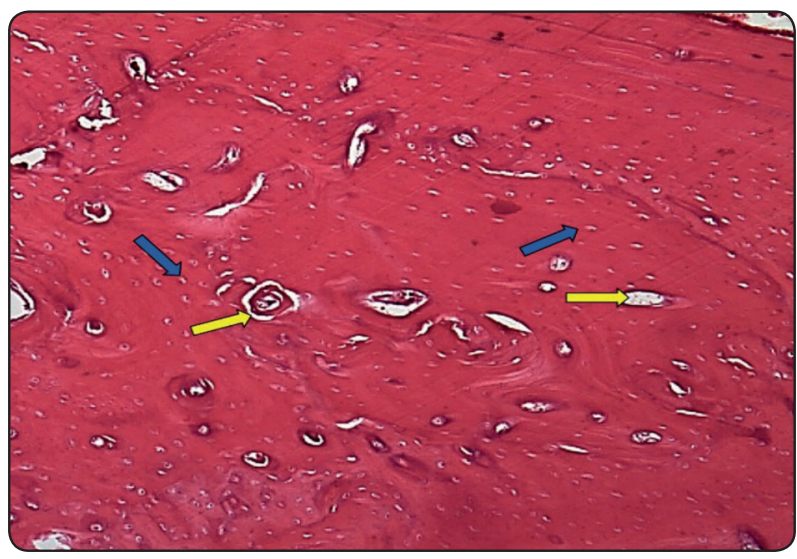

Fig. (1): A photomicrograph of cortical bone of control group (group I) showing well developed Haversian systems formed of Haversian canal (yellow arrows) and concentric lamellae of bone with well-developed osteocytes (blue arrows) (H\&E x 200).

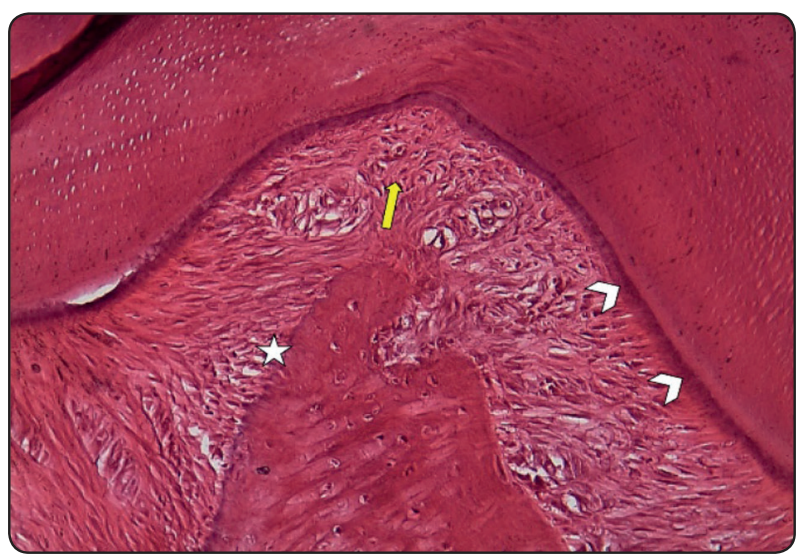

Fig. (3): A photomicrograph of control group showing interradicular group of periodontal ligaments of normal fibers and fibroblasts density radiating from the crest of the inter-radicular septum to cementum of the furcation of roots (arrows). Smooth surfaces of inter-radicular bone (star) and cementum (arrow heads) (H\&E x 200). decreasing the size of bone marrow cavities and no signs of fatty degeneration (Fig.9). Reorganization of oblique, inter-radicular and apical group of PDL, nearly smooth surfaces of adjacent alveolar bone and cementum were observed. Small sized areas of degeneration in some areas were evident (Figs.10, $11 \& 12)$

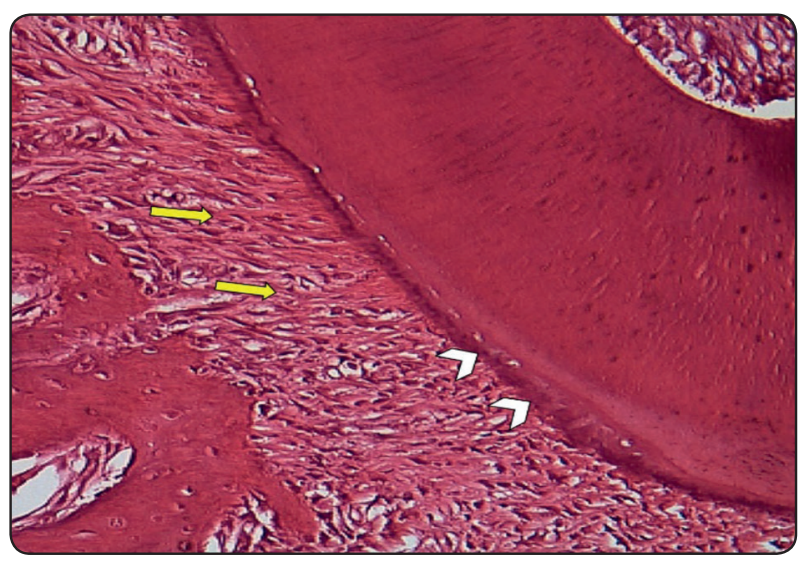

Fig. (2): A photomicrograph of control group showing proper orientation of oblique group of periodontal ligaments with normal density of fibroblasts and collagen fibers (arrows). Smooth cementum with normal thickness (arrow heads) (H\&E x 200).

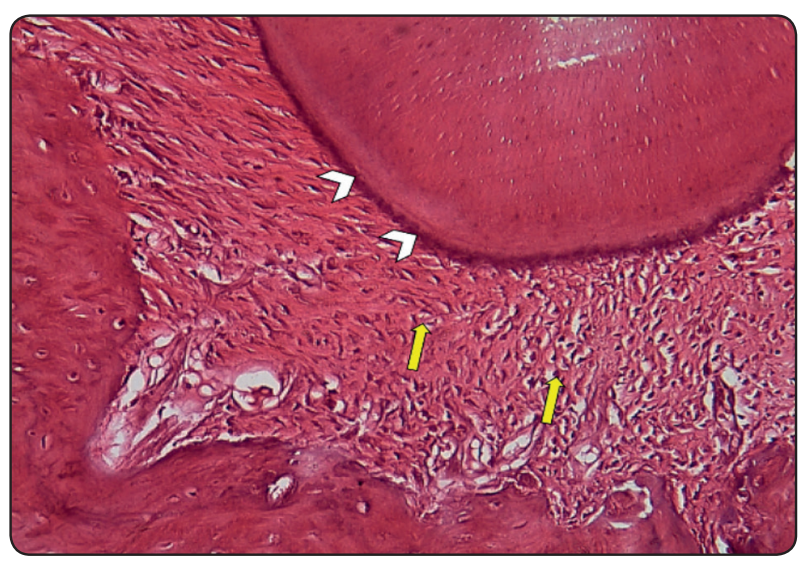

Fig. (4): A photomicrograph of control group showing apical group of periodontal ligaments of normal fibers and fibroblasts density and normally radiating from the cementum around the apex of the root to the fundus of the socket (arrows). Normal thickness of smooth cementum (arrow heads) (H\&E x 200). 


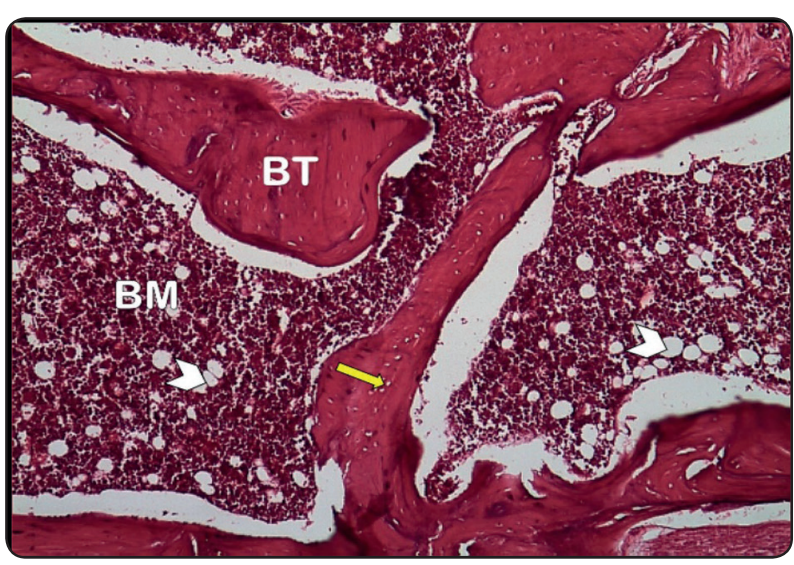

Fig. (5): A photomicrograph of old age group (group II) showing thin bone trabeculae (BT) and very wide bone marrow cavities (BM) with fatty degeneration (arrow heads). Some lacunae of osteocytes appeared empty (arrow) (H\&E x 200).

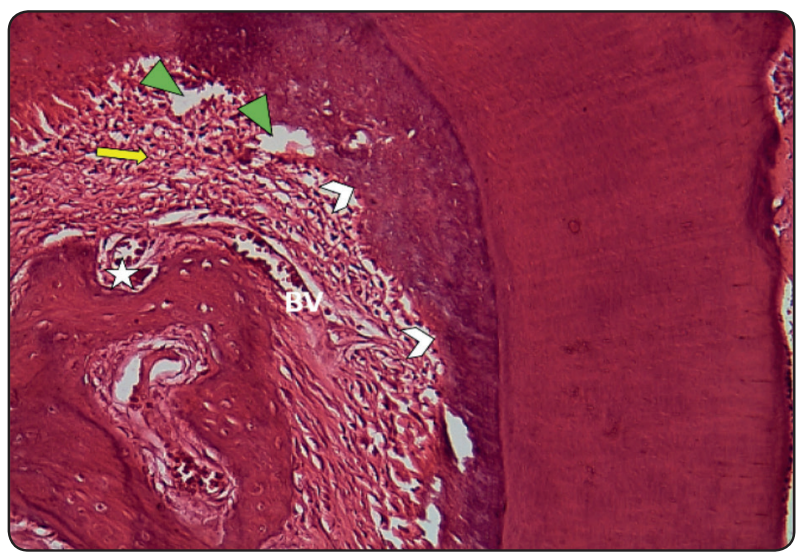

Fig. (7): A photomicrograph of group II showing disoriented fibers and fibroblasts of inter-radicular group (arrow) of PDL with areas of degeneration (triangle). Large sized blood vessels (BV), thickened irregular inter-radicular cementum (arrow heads) and irregular bone surface (star) (H\&E x 200).

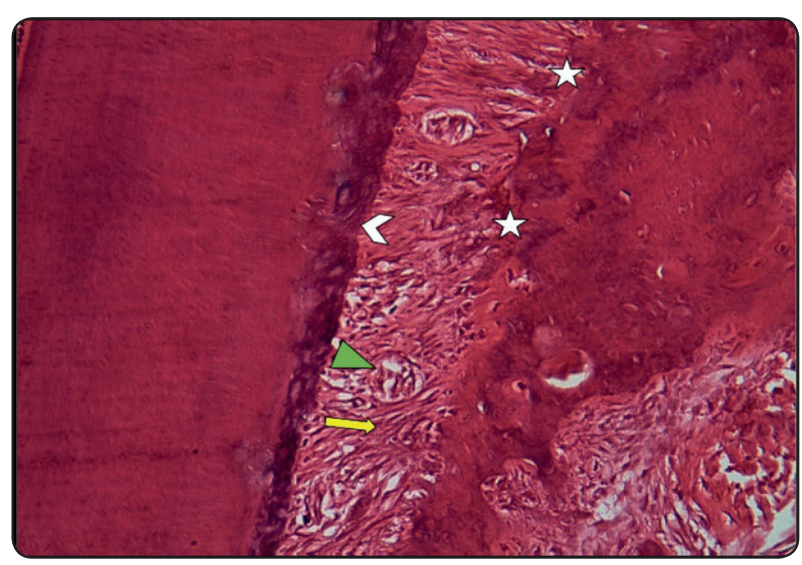

Fig. (6): A photomicrograph of group II showing disorganization of oblique fibers and fibroblasts of periodontal ligaments (arrow) with widening of interstitial spaces (triangle). Irregular and resorbed bone surface (stars) and cementum surface (arrow head) (H\&E x 200).

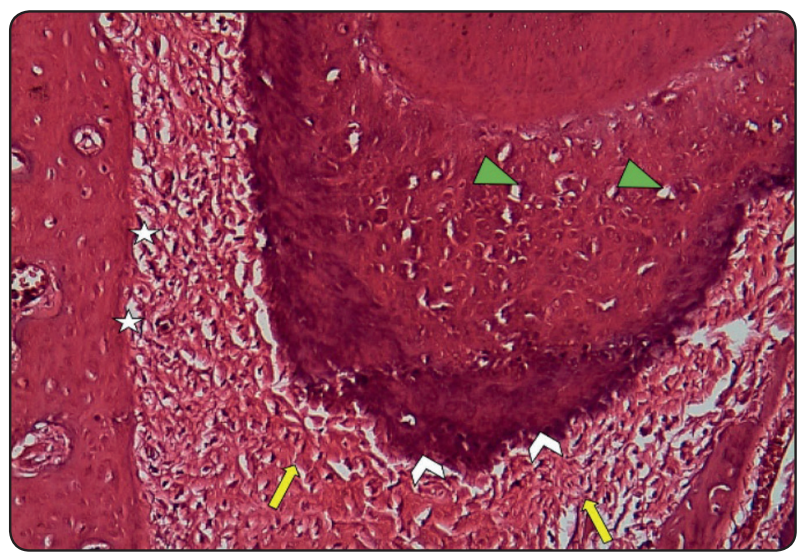

Fig. (8): A photomicrograph of group II showing abnormal disfigured fibers of apical group (arrows) of PDL, cementum hypercementosis (arrow heads), empty lacunae of cementocytes (triangles) and irregular bone surface (stars) (H\&E x 200). 


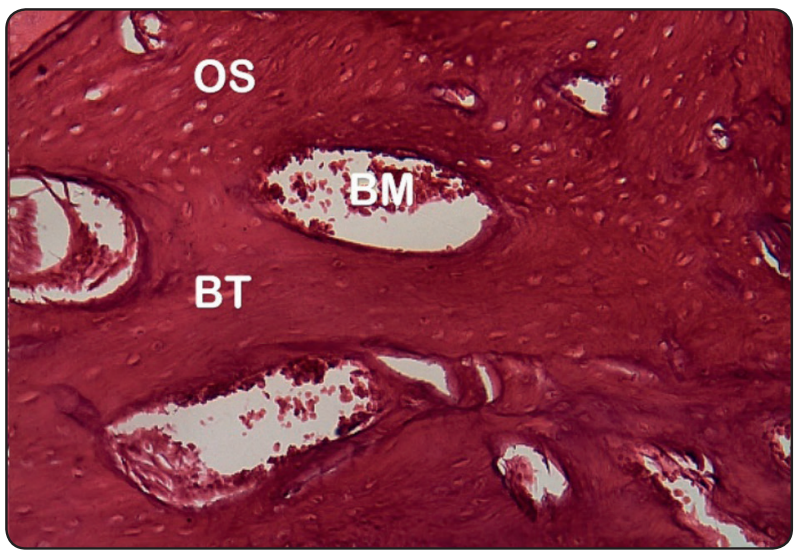

Fig. (9): A photomicrograph of old age group received nanosilver (group III) showing thick bone trabeculthe ae (BT) with numerous osteocytes (OS). Almost normal size of bone marrow cavities (BM). (H\&E x 200).

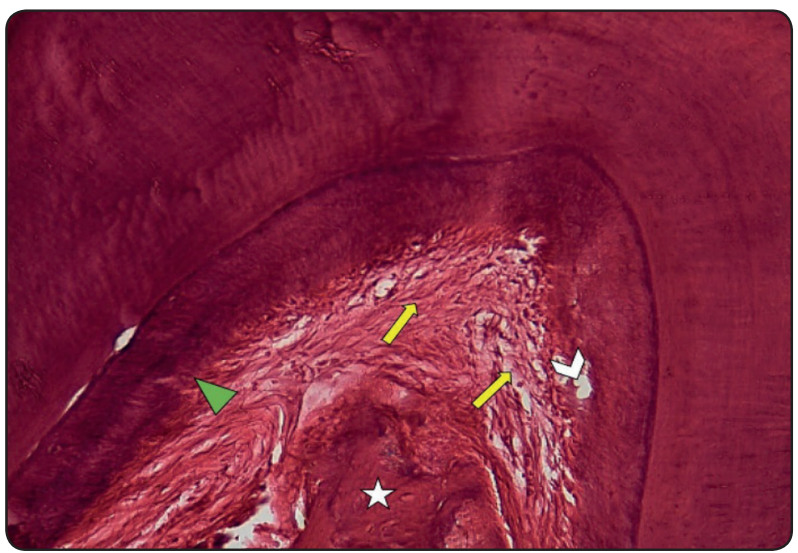

Fig. (11): A photomicrograph of group III showing reorganized fibers of inter-radicular group (arrows) of PDL with small sized areas of degeneration (arrow head), smooth surface of inter-radicular cementum (triangle) and intact inter-radicular bone (star) (H\&E x 200).

\section{DEXA results}

DEXA examination revealed, there was a reduction in mean of alveolar bone mineral density of old age (group II) compared with adult control (group I). Mean of alveolar bone mineral density in old group received silver nanoparticles (group III) increased as compared with group II but still less than that of group I. Statistical analysis of dual energy X-ray absorbometery (DEXA) measurements revealed statistical significant difference in alveolar bone mineral density between different groups by using ANOVA test (table I \& Fig.13).

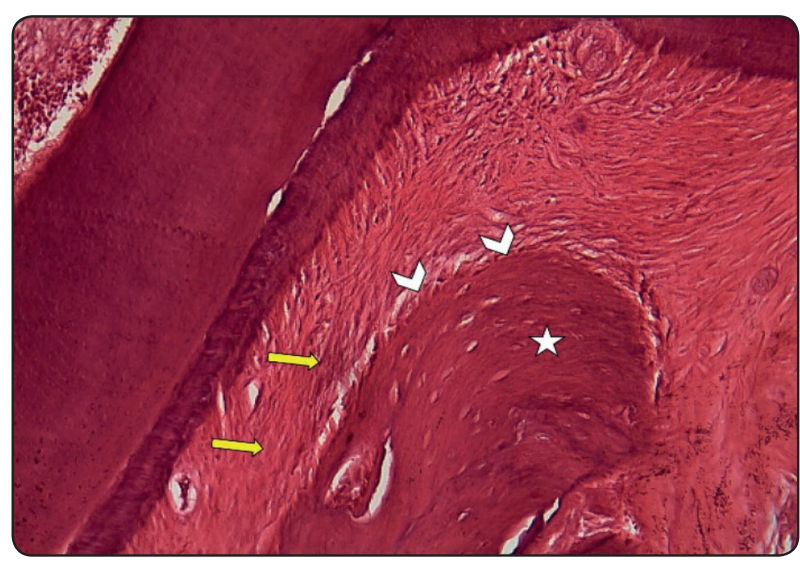

Fig.(10): A photomicrograph of group III showing reorganization of oblique fibers of PDL (arrows), normal density of collagen fibers, fibroblasts and occupying the normal width. Intact alveolar bone (star) with smooth surface (arrow heads) (H\&E x 200).

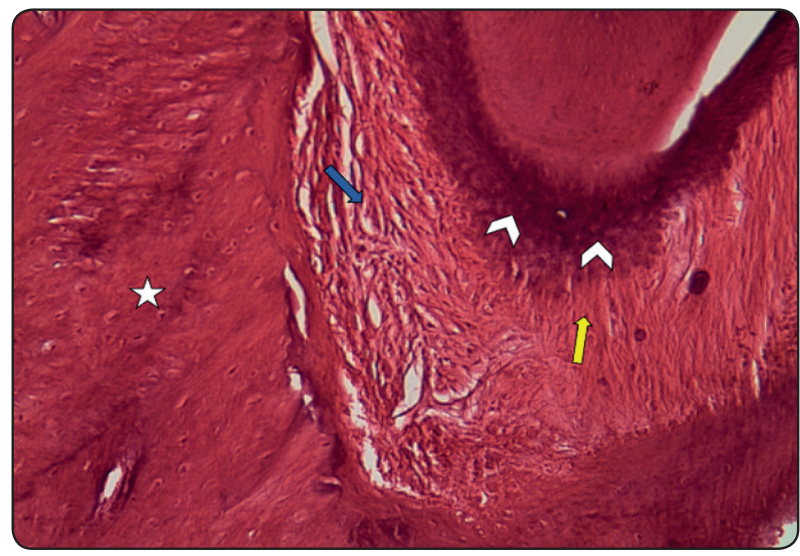

Fig. (12): A photomicrograph of group III showing nearly normal oriented fibers of apical group of PDL (yellow arrow) with small areas of dissociation (blue arrow). Medium thickness of cementum (arrow heads) and normal compact bone (star) (H\&E x 200).

TABLE (I) Showing the difference in mean alveolar bone mineral density between different groups using ANOVA test.

\begin{tabular}{|c|c|c|c|}
\hline \multirow{2}{*}{ Group } & \multicolumn{3}{|c|}{ Mean alveolar bone mineral density } \\
\cline { 2 - 2 } & Mean \pm SD & F -value & $p$-value \\
\hline Group I & $0.099 \pm 0.591$ & & \\
\hline Group II & $0.061 \pm 0.421$ & \multirow{2}{*}{16.35} & $<0.0001^{* *}$ \\
\hline Group III & $0.091 \pm 0.203$ & & \\
\hline
\end{tabular}

**Statistical significant difference $(p<0.0005)$ 


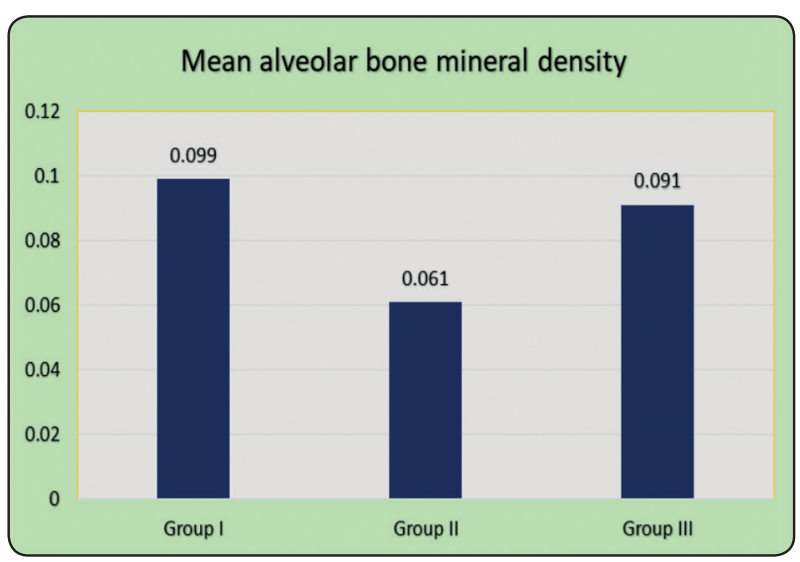

Fig. (13): A histogram representing the difference in mean alveolar bone mineral density between different groups.

\section{DISCUSSION}

The way of diagnosis, treatment, and prevention of various diseases in all aspects of human life was radically developed due to recent advances of nanotechnology. Silver nanoparticles (AgNPs) are broadly utilized in different fields and played a pivotal role especially in nanomedicine due to their unique physical and chemical properties ${ }^{(12)}$. Therefore, the present work was carried out to evaluate the possible biological effects of silver nanoparticles on age changes of the alveolar bone, cementum and periodontal ligaments of albino rats.

In materials and methods of the present study, we selected the chemical synthesis of nanosilver and a silver nitrate was used as a silver salt according to Tien et al., ${ }^{(13)}$ who reported that, the mostly used method for nanosilver synthesis is the chemical reduction method and the silver nitrate is the most frequently utilized silver salt due to its low cost and it is chemically stable compared to the other silver salts.

Light microscopic examination of $\mathrm{Hx} \& \mathrm{E}$ stained sections of the alveolar bone, cementum, periodontal ligaments of old age rats (group II) revealed that, group I appeared with its normal histological structures while in group II, alveolar bone showed thinning of bone trabeculae with some empty lacunae of osteocytes, widening of bone marrow cavities containing multiple fat cells and irregular resorbed bone surface. Irregular and resorbed cementum surface, hypercementosis and signs of degeneration as empty lacunae of cementocytes were evident. These results coincides with results obtained by Gomes et al., (14) and Locker et al., ${ }^{(15)}$ who found occurrence of irregularities on surfaces of alveolar bone and cementum in elderly patients. Apical portion of the roots showed increased cementum deposition. There is bone tissue change as the amount of mineralized material in both cortical and trabecular bone reduced. This change leads to decrease the bone resilience, and consequently increases the bone fragility. Commonly, there is overall bone porosity due to increased bone resorption and decreased bone formation. According to Bhaskar ${ }^{(16)}$, hypercementosis is the most common age changes of cementum. It is an abnormal thickening of cementum, and it may be diffuse or circumscribed.

The life cycle of cementocytes is typically characterized by aging and cell death. This could be explained by a rapid decrease in the accessibility of nutritional elements and inefficient disposal of waste products of the cementocytes .Therefor with age, cementum becomes acellular. In spite of remodeling of cementum occurs uncommon, but with age resorption at the cementum surface followed by cementum apposition is often seen, this might lead to uneven cementum surfaces ${ }^{(17)}$.

These result also were in agreement with Chan and Duque (18), Suva ${ }^{(19)}$ and Turgeman (20) who reported that, the cellular explanation of bone loss is multifactorial, with both decreases in bone deposition and increases in bone resorption ${ }^{(19)}$. Studies in elderly humans ${ }^{(18)}$ and mice ${ }^{(20)}$ have explained the decreases in bone formation due to a reduction in number of osteoblast progenitors, decreased proliferation, differentiation, and halflife. In aging bone, cellular apoptosis increased 
and it is more predominant in osteocytes, which are thought to act as the mechanosensors in bone. According to Patil and Patil ${ }^{(21)}$, in old age, anabolism decreases and there is a slight increase in catabolic processes. Production of matrix is further impaired by lack of proteins which are not either present in the diet in sufficient amount or are not completely utilized. Calcium deficiencies and negative calcium balance are frequent in the elderly people.

In the current work, light microscopic results of periodontal ligaments of group II showed that, disorganization and abnormal orientation of collagen fibers of oblique, interradicular and apical groups of PDL, decreased density of collagen fibers and fibroblasts. Amalgamation and dissociation of collagen fibers with multiple areas of degeneration. These results could be explained by Russell and Suter ${ }^{(22)}$, Li and Schellhorn ${ }^{(23)}$ who stated that, by aging, collagen fiber in PDL damaged and collagen synthesis might be altered as a consequence of reduction of ascorbic acid, which presents decresed plasmatic levels as a result of the high level of oxidative stress among other factors. Moreover, the harmful impacts of free radicals are one of the leading factors of cell aging ${ }^{(24)}$. According to Matsuzaka et al., ${ }^{(25)}$ in aged rats, the fibroblasts of PDL proliferated slowly as compared with young rats. In the work of Goseki et al., ${ }^{(26)}$ reduction of secretion of collagen was observed through aging, and at that stage, the catabolism in the fibroblast dominate over the anabolism. In another study, aging does not impair collagen formation, however, delays tissue maturation ${ }^{(27)}$.

The gradual retrogradation of the immune system which is called as Immunosenescence caused by natural aging ${ }^{(28)}$. Aging is a complicated, continually, and slowly developing process that progressively affects most if not all organs of the organism, leading to both qualitative and quantitative changes such as abnormal functions and morphological or structural changes of these organs ${ }^{(29)}$.

Regarding the histological results of old age received Ag NPs (group III), smooth surface of cementum and bone with no signs of degeneration were observed. Cementum appeared nearly of normal thickness. Bone trabeculae were thicker and bone marrow cavities appeared narrower than group II. New periodontal ligament fibers formation, reorganization, and reorientation all groups of PDL with normal density of collagen fibers and fibroblasts were noted. Our results were supported by Saravanan et al., ${ }^{(30)}$ and $\mathrm{Wu}$ et al. ${ }^{(31)}$ who performed their study to evaluate the usage of silver nanoparticles in dental applications for the structural and surface modification of bone grafts and membranes. They showed that the Ag-nHA/TiO2/PA66 developed a highly porous antimicrobial scaffold that could be efficient for the continuous Ag+ release at the boneimplant interface. Accordingly, these scaffolds would supply excellent mechanical backing and preservation for cells to adhere, migrate and proliferate, and bode of clinical applications of Ag NPs in repair, regeneration and augmentation of bone.

Reorganization of periodontal ligaments could be explained by Liu et al., ${ }^{(32)}$ as they demonstrated that AgNPs could be responsible for induction of fibroblasts proliferation and maturation. Liu et al., ${ }^{(33)}$ detected a plentiful amount of collagen in wound healing of skin by AgNPs. Moreover, the collagen fibrils in AgNPs-treated healed skin demonstrated more organization and compactness of its alignment, and it appeared closely resemble to that of the normal skin.

We suggested that, the improvement of histological results in group III might be due to multiple biological roles of AgNPs.

This suggestion is supported by those reported by El-Badawyqn et al., ${ }^{(34)}$ and Zhong et al., ${ }^{(35)}$ who stated that AgNPs became the most interesting field in biomedical implications, due to their efficient 
activity against bacteria, fungi, viruses, and inflammation. Also Nadworny et al., ${ }^{(36)}$ revealed that, in pocrine model of contact dermatitis, healing process was greatly improved as a result of direct anti-inflammatory effects of AgNPs. Lansdown, (37) and Castellano et al., (38) explained that, the antibacterial activity of silver ions might be due to its high reactivity as it binds to tissue proteins leading to structural alterations in the cell wall and nuclear membrane of the bacteria and consequently cell deformity and death. Furthermore, silver binds to nucleic and ribonucleic acids of the bacteria by denaturing and preventing bacterial reproduction.

The capability of $\mathrm{Ag}$ NPs to decrease the cytokine release and matrix metalloproteinases, ${ }^{(39)}$, reduces lymphocytes and mast cells infiltration ${ }^{(40)}$ and lead to apoptosis in inflammatory cells ${ }^{(39,41)}$ might explain their anti-inflammatory activities.

Concerning DEXA examination and using analysis of variance (ANOVA) test, the difference in mean of bone mineral density between the groups was found to be statistically significant, BMD decreased in old age group compared with control and enhanced in old age group received silver nanoparticles as compared with old age group but still less than control group. This finding coincided with other researchers who reported that, by aging, the cortical bone mineral density decreases in intact female rats start in the lumbar vertebrae at the age about 15 months and in the proximal tibial metaphysis at the age about 12 months ${ }^{(42)}$. Results from the evaluation of BMD in rat proximal femur by DEXA reported an age-associated decrease of BMD in old age animals ${ }^{(43 \& 44)}$. The enhanced bone density in group III could be attributed to receiving silver nanoparticles, this suggestion was in accordance with Li et al., ${ }^{(45)}$ who demonstrated the positive effects of Ag NPs in guided bone regeneration. In addition, all our histological results and its explanations confirmed our DEXA examination results.

\section{CONCLUSION}

Oral administration of silver nanoparticles could improve the histological age changes of the alveolar bone, cementum and periodontal ligaments with enhancement of bone mineral density of the alveolar bone. density in the test group could be attributed to the addition of Ag NPs to the grafted materigh. This suggestion was in accordance with $\mathrm{Li}$ et al who demonstrated the positive effects of Ag NPs in the guided bone regeneration (GBR)

\section{REFERENCES}

1. Balantrapu K and Goia D. Silver nanoparticles for printable electronics and biological applications. J Mater Res 2009; 24: 2828-2836.

2. Silva, G.A. Introduction to nanotechnology and its applications to medicine. Surg. Neurol. 2004, 61, 216-220.

3. Abbas B, Roghayeh G, Mohammad ZK, Ahmad S. Antimicrobial activity of acrylic resins with in-situ generated nanosilver on cariogenic planktonic and biofilm bacteria. Int Res J Biol Sci 2014; 3:38-46.

4. Carlson, C.; Hussain, S.M.; Schrand, A.M.; BraydichStolle, L.K.; Hess, K.L.; Jones, R.L.; Schlager, J.J. Unique cellular interaction of silver nanoparticles: Size-dependent generation of reactive oxygen species. J. Phys. Chem. B 2008, 112, 13608-13619.

5. Tiwari DK, Jin T, Behari J. Dose-dependent in-vivo toxicity assessment of silver nanoparticle in Wistar rats. Toxicol Mech Methods. 2011; 21:13-24.

6. Atiyeh BS, Costagliola M, Hayek SN, Dipo SA. Effect of silver on burn wound infection control and healing: review of the literature. Burns. 2007; 33:139-148.

7. Fischer HC \& Chan WCW., 2007 Nanotoxicity: the growing need for in vivo study Curr Opin Biotechnol 18, $565-571$.

8. Stensberg MC, Wei Q, McLamore ES, Porterfield D M, Wei A\& Sepúlveda MS., 2011 Toxicological studies on silver nanoparticles: challenges and opportunities in assessment, monitoring and imaging. Nanomedicine (Lond) 6(5)879-98.

9. R. Guiglia1, A. Musciotto1, D. Compilato1, M. Procaccini2, L. Lo Russo3, D. Ciavarella3, L. Lo Muzio3, V. Cannone 
4, I. Pepe4, M. D’Angelo1 and G. Campisi1,2010 Aging and Oral Health: Effects in Hard and Soft Tissues. Current Pharmaceutical Design, 16, 619-630.

10. Corrêa da Silva, SR; Fernandes, RAC. Auto Percepção das Condições de Saúde Bucal por Idosos. Revista Saúde Publica, v.35, p.349-55, 2001.

11. Turkevich J, Stevenson PC \& Hillier J., 1951 A study of the nucleation and growth processes in the synthesis of colloidal gold. Discuss Faraday Soc.11, 55-75.

12. Gurunathan, S.; Park, J.H.; Han, J.W.; Kim, J.H. Comparative assessment of the apoptotic potential of silver nanoparticles synthesized by Bacillus tequilensis and Calocybe indica in MDA-MB-231 human breast cancer cells: Targeting p53 for anticancer therapy. Int. J. Nanomed. 2015, 10, 4203-422.

13 Tien DC, Liao CY, Huang JC, et al. Novel technique for preparing a nano-silver water suspension by the arcdischarge method. Reviews on Advanced Materials Science. 2008;18:750-756. (preparation of nano silver)

14. Gomes, SGF; Meloto, CB; Custodio, W; Rizzatti-Barbosa, CM. Aging and the periodontium. Braz J Oral Sci., v. 9, p. 1-6, 2010

15. Locker D, Slade GD, Murray H. Epidemiology of periodontal disease among older adults: a review. Periodontol., 2000, v.16, p.16-33, 1998.

16. Bhaskar SN, Orban's Oral Histology and thEmbryology, 11 edition, Elsevier India Pvt. Ltd.; 1997; 180-333.

17. Van der Velden U. Effect of age on the periodontium. J Clin Periodontol. 1984; 11: 281294.

18. Chan GK and Duque G. Age-related bone loss: old bone, new facts. Gerontology 48:62-71, 2002.

19. Suva LJ, Gaddy D, Perrien DS, Thomas RL, and Findlay DM. Regulation of bone mass by mechanical loading: microarchitecture and genetics. Curr Osteoporos Rep 3:4651, 2005.

20. Turgeman G, Zilberman Y, Zhou S, Kelly P, Moutsatsos IK, Kharode YP, Borella LE, Bex FJ, Komm BS, Bodine PV, and Gazit D. Systemically administered rhBMP-2 promotes MSC activity and reverses bone and cartilage loss in osteopenic mice. JCell Biochem 86:461-474, 2002.

21. Patil MS, Patil SB. Geriatric patient - psychological and emotional considerations during dental treatment. Gerodontology 2009; 26:72-7.
22. Russell Rm And Suter M. 1993. Vitamin requirements of elderly people: an update. Am J Clin Nutr 58: 4-14.

23. Li Y and Schellhorn HE. 2007. New Developments and Novel Therapeutic Perspectives for Vitamin C. J Nutr 137: 2171-2184.

24. Azulay Mm, Lacerda Cam, Perez A, Filgueira Al And Cuzzi T. 2003. Vitamin C. An Bras Dermatol 78: 265-274.

25. Matsuzaka K ET AL. 2007. Age-related Differences in Expression of Vascular Endothelial Growth Factor by Periodontal Ligament Cells in vitro. Bull Tokyo Dent Coll 48: 143-146.

26. Goseki T, Shimizu N, Iwasawaa T, Takiguchib H And Abikob Y. 1996. Effects of in vitro cellular aging on alkaline phosphatase, cathepsin activities and collagen secretion of human periodontal ligament derived cells. Mech Aging Dev 91: 171-183.

27. Biondo-Simões Mlp, Westphal Vl, Paula Jb, Borsato Ks And Noronha L. 2005. Collagen synthesis after the implantation of polypropylene nets in the abdominal wall of young and old rats. Acta Cir Bras 20: 300-304.

28. Franceschi C, Valensin S, Fagnoni F, Barbi C, Bonafè M. Biomarkers of immunosenescence within an evolutionary perspective: the challenge of heterogeneity and the role of antigenic load. Exp Gerontol. 1999; 34: 911-921.

29. Malaguarnera L, Ferlito L, Imbesi RM, Gulizia GS, Di Mauro S, Maugeri D, Malaguarnera M, Messina A. Immunosenescence: a review. Arch Gerontol Geriatr 2001; 32:1-14

30. S. Saravanan, S. Nethala, S. Pattnaik, A. Tripathi, A. Moorthi, and N. Selvamurugan, "Preparation, characterization and antimicrobial activity of a bio-composite scaffold containing chitosan/nano-hydroxyapatite/nano-silver for bone tissue engineering," International Journal of Biological Macromolecules, vol. 49, no. 2, pp. 188-193, 2011.

31. X. Wu, J. Li, L. Wang, D. Huang, Y. Zuo, and Y. Li, “The release properties of silver ions from Ag-nHA/TiO2/PA66 antimicrobial composite scaffolds," Biomedical Materials, vol. 5, no. 4, Article ID 044105, 2010.

32. Liu X, L, H, L, C, C, T, W. (2010): Silver nanoparticles mediate differential responses in keratinocytes and fibroblasts during skin wound healing; 5: 468-475. 45.

33. Liu X. (2013): The role of AgNP on skin wound healing tissue remodeling and their potential cytotoxicity PHD. The University of Hong Kong (Pokfulam, Hong Kong). 
34. El-Badawy A, Feldhake D, Venkatapathy R. State of the Science Literature Review: Everything Nanosilver and More. Washington, DC: US Environmental Protection Agency; 2010.

35. Zhong W, Xing MM, Maibach HI. Nanofibrous materials for wound care. Cutan Ocul Toxicol. 2010;29(3):143-152.

36. Nadworny P, Wang J, Tredget E, Burrell R. (2008): Antiinflammatory activity of nanocrystalline silver in a porcine contact dermatitis model. Nanomedicine: Nanotechnology, Biology and Medicine; 4: 241-251.

37. Landsdown A. (2002): Silver I: its antibacterial properties and mechanism of action. JWound Care;11:125-138. 43.

38. Castellano J, Shafii S, Ko F, Donate G, Wright T, Mannari R, P, S, R. Comparative evaluation of silvercontaining antimicrobial dressings and drugs. Int Wound $\mathrm{J}$ 2007; 4(2):114-122.

39. Sibbald RG, Contreras-Ruiz J, Coutts P, Fierheller M, Rothman A, Woo K. Bacteriology, inflammation, and healing: a study of nanocrystalline silver dressings in chronic venous leg ulcers. Adv Skin Wound Care. 2007; 20(10):549-558.
40. Nadworny PL, Wang J, Tredget EE, Burrell RE. Anti-inflammatory activity of nanocrystalline silver in a porcine contact dermatitis model. Nanomedicine. 2008;4(3):241-251.

41. Shin SH, Ye MK. The effect of nano-silver on allergic rhinitis model in mice. Clin Exp Otorhinolaryngol. 2012; 5(4):222-227.

42. Fukuda S, Iida H. 2004. Age-related changes in bone mineral density, cross-sectional area, and the strength of long bones in the hind limbs and the first lumbar vertebra in female Wistar rats. J Vet Med Sci 66:755-760.

43. World Health Organization (WHO). Assessment of osteoporosis at the primary health care level. Summary Report of a WHO Scientific Group 2007; WHO: Geneva, Switzerland.

44. Kanis, J.A.; on behalf of the World Health Organization Scientific Group. Assessment of Osteoporosis at the Primary Health-Care Level; WHO Collaborating Centre, University of Sheffield: Sheffield, UK, 2007.

45. Li J, Zuo Y, Man Y, Mo A, Huang C, Liu M, Jansen JA, Li Y. Fabrication and biocompatibility of an antimicrobial composite membrane with an asymmetric porous structure. J Biomater Sci Polym Ed. 2012; 23: 81-96. 